La Suisse et la zone euro : votre monnaie, notre problème?

La possibilité d'un ancrage de jure

$\mathbf{e}$

Cyriac Guillaumin, Guillaume Vallet

h

$\mathbf{e}$

$\boldsymbol{r}$

C

h

$\mathbf{e}$

Cahier de recherche du Creg, $\mathrm{n}^{\circ} 2011.03$

Juillet 2011

$\mathbf{u}$

C

i.

E

CREG - Centre de Recherche en Économie de Grenoble Faculté d'Économie de Grenoble - UPMF, BP 47, 38040 Grenoble Cedex 9 Tél : +33 (0)4 76825692 ; E-mail : creg@upmf-grenoble.fr http://creg.upmf-grenoble.frl 



\title{
La Suisse et la zone euro : votre monnaie, notre problème ? \\ La possibilité d'un ancrage de jure
}

\author{
Cyriac Guillaumin $^{*} \quad$ Guillaume Vallet ${ }^{* *}$
}

Résumé : Avec les récentes tensions au sein de la zone euro, le franc suisse s'est fortement apprécié face à l'euro, jouant un rôle de monnaie refuge internationale. Même si la Suisse n'est pas membre de l'Union européenne (UE), son degré élevé d'intégration de facto à celleci fait qu'une telle appréciation se transmet à l'économie réelle. Ainsi, si l'indépendance et l'autonomie monétaires officielles de la Suisse en Europe ont des avantages, elles induisent aussi des coûts non négligeables, notamment liés au statut particulier du franc suisse. Nous étudions dans cet article l'opportunité et la viabilité pour la Suisse d'une intégration monétaire «médiane » à l'UE qui permettrait de desserrer cette contrainte extérieure du taux de change, à savoir un ancrage de jure du franc suisse à l'euro. En nous intéressant plus précisément aux origines des fluctuations du taux de change du franc suisse à l'aide d'un modèle VAR structurel, nous mettons en évidence que l'ancrage serait viable pour la Suisse mais au prix d'une perte d'autonomie monétaire significative.

Mots-clefs : Suisse, Union européenne, régime de change, ancrage officiel, modèle VAR structurel.

Classification JEL : E42, E58, F15, F31.

\footnotetext{
*CREG, université de Grenoble. Courriel : cyriac.guillaumin@upmf-grenoble.fr.

** Université de Grenoble. Courriel : guillaume.vallet@upmf-grenoble.fr.

Correspondance : Guillaume Vallet, Université de Grenoble, 1241 rue des Résidences, Domaine universitaire, BP 47, 38040 Grenoble cedex 9, France. Courriel : guillaume.vallet@upmf-grenoble.fr. Tel : +33 (0)4 76825765 .
} 


\section{Introduction}

La crise financière, débutée en 2007, puis économique, à partir de 2008, s'est accompagnée de nombreuses «turbulences» sur les marchés des changes internationaux, se traduisant notamment par l'appréciation soudaine et de grande ampleur de certaines monnaies. Si l'évolution des parités nominales de l'euro et du yen vis-à-vis du dollar est illustrative d'un tel phénomène, le cas du cours du franc suisse est moins souvent évoqué alors qu'il est particulièrement représentatif. En effet, jouant historiquement une fonction de valeur refuge internationale en cas de crise internationale, le franc suisse tend à s'apprécier fortement et rapidement vis-à-vis des principales devises internationales. Ce facteur est renforcé par le degré élevé d'ouverture économique et financière du pays.

Mais plus que l'appréciation de la monnaie, c'est son rythme et surtout les paliers franchis qui interpellent, surtout vis-à-vis de l'euro, devise des principaux partenaires helvétiques. Or cette tendance pose, à nouveau, en Suisse, la question de la pertinence de la position politique, économique et monétaire du pays en Europe. En effet, pourtant située au cœur de la « dorsale européenne », et entourée par les pays membres de l'Union économique et monétaire (UEM), la Suisse ne fait partie ni de l'Union européenne (UE) ni de la zone euro. Il s'agit d'une volonté politique ancienne qui se retrouve dès les débuts de la construction européenne, celleci étant présentée comme conduisant à une remise en cause de spécificités nationales politiques (fédéralisme, neutralité) comme économiques (secret bancaire, fiscalité attractive). Le problème pour la Suisse est qu'étant fortement intégrée de facto à l'UE et à la zone euro, le pays subit dans une grande ampleur les fluctuations de la parité franc suisse/euro, ce qui pénalise les secteurs industriels exportateurs et accentue les pressions déflationnistes qui affectent actuellement les économies de l'UEM. Ce facteur est aggravé lorsque le franc suisse exerce sa fonction de valeur refuge puisque, dans ce cas, l'appréciation ne correspond pas aux fondamentaux de l'économie. D'où de fréquentes et massives interventions de la Banque nationale Suisse (BNS) sur le marché des changes pour tenter de contrecarrer cette tendance, mais qui sont critiquées et même inefficaces, en particulier parce qu'elles sont non coordonnées avec la Banque centrale européenne (BCE).

De ce fait, une telle position n'est-elle pas a priori fragile et vouée à terme à l'échec ? Si la Suisse maintient son refus politique d'adhérer à l'UE à court terme, un «arrangement monétaire médian » ne pourrait-il pas être envisagé avec les autorités monétaires et politiques de l'UE? Plus précisément, compte tenu de la particularité de la position suisse « d'intégration non adhésive », la mise en place d'un ancrage officiel de la monnaie nationale 
à l'euro ne pourrait-elle pas constituer une «dépendance monétaire libératrice » pour la Suisse ? Nous tentons de répondre à ces questions au cours de cet article, qui est organisé de la façon suivante : la section 1 précise la position particulière de la Suisse à l'égard de l'UE, à la fois fortement intégrée à cette zone sans en être membre. Dans la section 2, nous expliquons alors pourquoi cette «intégration non adhésive » expose potentiellement le pays aux variations de la parité franc suisse/euro. Etant donné cette contrainte extérieure qui pèse sur le pays et compte tenu de la volonté politique helvétique de ne pas adhérer à l'UE, la section 3 détaille les avantages et les inconvénients d'un ancrage fixe du franc suisse à l'euro comme «dépendance monétaire libératrice ». La section 4 s'intéresse aux origines des fluctuations du taux de change du franc suisse à l'aide d'un modèle VAR structurel. Enfin, la section 5 conclut.

\section{La Suisse dans le « maelström » européen}

Dans cette partie, nous montrons que même si la Suisse est intégrée mais non membre de l'UE depuis plus de 50 ans, le pays est malgré tout fortement dépendant de la dynamique économique et financière de l'UE. Or ce degré élevé d'intégration et cette dépendance à l'égard de l'UE posent à son tour la question du degré d'exposition aux variations de la parité franc suisse/euro.

\subsection{La Suisse, passager toléré de l'Union européenne}

Si la Suisse est au «cœur » de l'Europe sur le plan géographique, elle ne fait pas partie de l'UE et de la zone euro. Dès les débuts de la construction européenne, la Suisse a tenu à prendre ses distances vis-à-vis de l'intégration économique et monétaire européenne, pour des raisons politiques liée aux délégations de souveraineté aux organes supranationaux de l'UE, mais surtout économiques et financières. En effet, si la Suisse dépend de longue date des marchés européens ${ }^{1}$, elle est ouverte aux échanges extérieurs extra-européens. Son taux d'ouverture ${ }^{2}$, évalué à $45 \%$ du PIB, inclut non seulement les pays de l'UE, mais aussi les pays asiatiques et surtout les États-Unis. Cela signifie que d'un point de vue économique, le pays est «entre deux mondes ». Ces derniers sont certes d'une importance respective inégale, mais ils montrent en tout cas que la situation économique suisse ne peut pas être pensée dans le cadre d'une dépendance unique à l'égard des marchés européens. De même, la Suisse est une

\footnotetext{
${ }^{1}$ Au moment de la signature du Traité de Paris (1951), 75\% des importations suisses de charbon, d'acier et de fer avaient pour origine les six pays de la CECA.

${ }^{2}$ Soit la moyenne du total des exportations et des importations en pourcentage du PIB.
} 
place financière européenne importante en dehors de la zone euro, dont les opérations avec des non-résidents sont massives. En lien également avec une monnaie internationalement reconnue, le pays est parvenu à construire un avantage comparatif prépondérant dans les domaines bancaire et financier (10\% du PIB). Plus particulièrement, la Suisse met en avant l'abondance des capitaux circulant sur son territoire qui induit en comparaison internationale un «bonus » de taux d'intérêt, qui pourrait disparaître en cas d'une adhésion à l'UEM, tout comme le « fameux » secret bancaire.

La posture suisse historique et actuelle en matière d'intégration européenne dénote non seulement une différence de degré, mais aussi une différence de nature par rapport à celle suivie par les pays aujourd'hui membres. Cela signifie que les différentes relations nouées avec l'UE doivent être perçues comme permettant non pas de se rapprocher de l'adhésion, mais au contraire de s'en éloigner. Ainsi, la Suisse se retrouve entre une situation « d'adhésion improbable » et de « marginalisation impossible » (Schwok, 2006).

C'est pourquoi, si la Suisse a négocié avec l'UE des accords de rapprochement, leur nature et leur finalité divergent de ceux noués par l'UE avec d'autres pays tiers, considérés au contraire souvent comme une étape intermédiaire avant l'adhésion. C'est le cas des accords de libreéchange pour les biens industriels et certains produits agricoles non transformés de 1972, mais surtout de ceux créant l'Espace Economique Européen (EEE), signés par les dirigeants politiques helvétiques en 1991, mais rejetés en 1992 par voie référendaire ${ }^{3}$ par la population suisse, car perçus par les citoyens comme un «sas » en direction de l'adhésion à l'UE. Ainsi, plus qu'illustrant un véritable revirement stratégique et rationnel des autorités helvétiques, cet exemple «malheureux » renforce au contraire l'idée d'une volonté suisse de ne pas adhérer intégralement à l'UE pour préserver certaines spécificités.

D'où la possibilité bienvenue de parvenir avec l'UE à un arrangement satisfaisant les deux parties avec la signature en 1999 de deux «paquets » d'accords bilatéraux portant sur un certain nombre de domaines ${ }^{4}$, et aux implications économiques diverses, qui régissent toujours aujourd'hui les relations entre les deux espaces sur le plan institutionnel. Certes, les accords bilatéraux constituent une modalité d'intégration qui comporte des désavantages pour la Suisse, du moins des contraintes : accès partiel au marché intérieur européen (entre $60 \%$ et

\footnotetext{
${ }^{3}$ Comme l'oblige la Constitution suisse pour les traités internationaux.

${ }^{4}$ Les "bilatérales I" signées le 21 juin 1999 concernent le transport aérien, l'ouverture des marchés publics, la participation suisse aux programmes de recherche communautaire, l'agriculture, l'abolition des obstacles techniques au commerce, le transport terrestre et le libre établissement des personnes. Les "bilatérales II", signées le 26 octobre 2004, traitent de la fiscalité de l'épargne, la lutte contre la fraude, les accords Schengen/Dublin, les produits agricoles transformés, la statistique, les pensions des fonctionnaires retraités de l'UE résidants en Suisse, l'environnement, la participation suisse au programme MEDIA et enfin l'éducation, la formation professionnelle et la jeunesse.
} 
80\% selon Ganser et alii, 2003), "clause guillotine », place seulement consultative et non décisionnelle pour la Suisse, intégration de la fiscalité de l'épargne dans les accords en particulier.

Toutefois, cette modalité d'intégration présente aussi de nombreux avantages pour le pays : renforcement et institutionnalisation de la participation helvétique au marché intérieur européen, participation au budget européen inférieure à celle liée à une adhésion. C'est pourquoi les milieux politiques et économiques helvétiques affirment que cette voie est actuellement la meilleure modalité d'intégration économique européenne de la Suisse, et elle doit être poursuivie à l'avenir.

Plus qu'un passager clandestin ne participant pas au fonctionnement d'un bien collectif, la Suisse apparaît alors davantage comme un «passager toléré » par l'UE : au nom de ses relations économiques de voisinage, l'UE accepte cette modalité d'intégration car il en va aussi de ses intérêts (élargissement des marchés, participation helvétique au budget communautaire,...). Du point de vue suisse en tout cas, ces accords lui permettent officiellement de préserver les conditions cadres de son économie, en particulier l'autonomie budgétaire et monétaire.

\subsection{Une dépendance économique et financière helvétique élevée}

Malgré sa volonté politique d'intégration institutionnelle «minimale », la Suisse est largement intégrée de fait à l'UE d'un point de vue économique. Nous qualifions cette

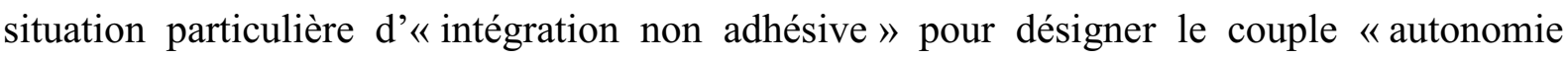
politique et forte intégration économique de fait ». Concernant ce dernier point, la Suisse et l'UE se situant dans une «zone naturelle d'échange $»^{5}$ ont, par conséquent, des échanges commerciaux réciproques intenses (graphique 1).

\footnotetext{
${ }^{5}$ Une telle zone est définie par Krugman (1991) comme une zone à l'intérieur de laquelle, en l'absence de barrières aux échanges et d'accords de préférence, les échanges seraient plus intenses qu'avec le reste du monde.
} 


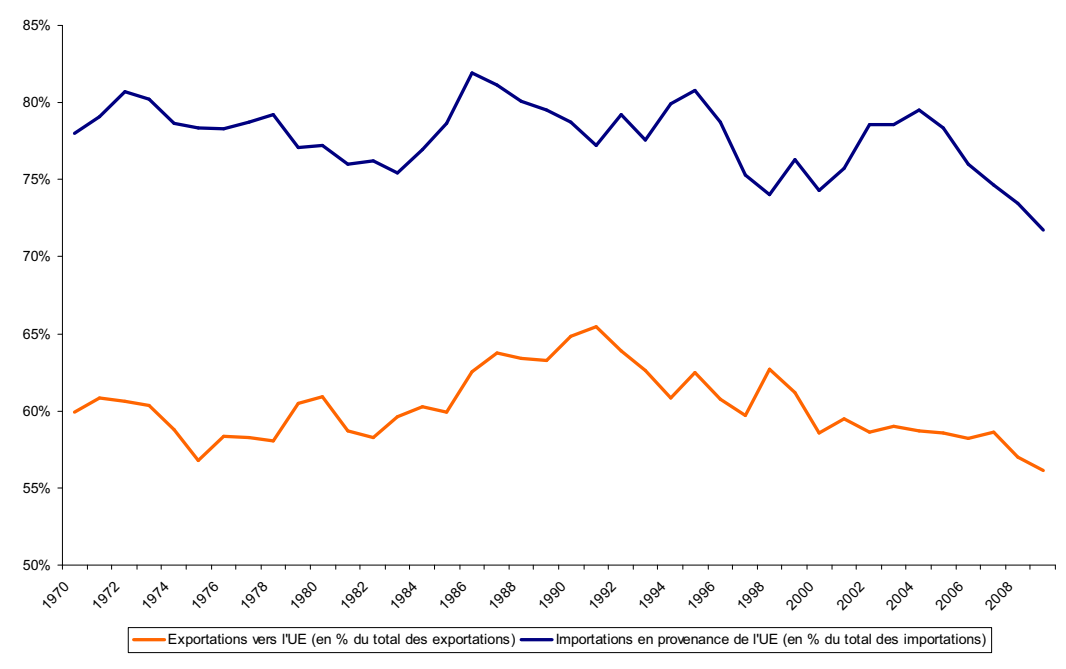

Graphique 1 : le commerce entre la Suisse et l'Union européenne. Source : calculs des auteurs à partir de données CHELEM (Cepii).

Mais compte tenu du degré élevé d'ouverture et de la taille relative de la Suisse, la dépendance est asymétrique: la Suisse dépend plus fortement du dynamisme de ses économies géographiquement voisines que l'inverse. Les tableaux 1 et 2 nous le montrent.

Tableau 1 : dépendance commerciale de la Suisse (en \%)

\begin{tabular}{ccccc}
\hline \hline & 1995 & 2000 & 2005 & 2009 \\
\hline \hline France & 10,78 & 10,01 & 9,51 & 8,54 \\
Allemagne & 29,27 & 25,58 & 26,09 & 26,37 \\
Italie & 9,17 & 8,51 & 9,59 & 9,73 \\
Espagne & 1,67 & 1,97 & 3,14 & 2,27 \\
Portugal & 0,59 & 0,39 & 0,34 & 0,30 \\
UE 15 & 71,74 & 66,71 & 68,49 & 63,94 \\
UE 27 & 73,34 & 68,82 & 71,36 & 66,96 \\
Zone euro & 63,77 & 59,61 & 61,95 & 59,25 \\
\hline \hline
\end{tabular}

Note : la dépendance commerciale se définit par le rapport entre la somme des exportations et des importations de la Suisse avec son partenaire et la somme des exportations et des importations totales de la Suisse. Source : calculs des auteurs à partir de données CHELEM (Cepii). 
Tableau 2 : intensité commerciale

\begin{tabular}{ccccc}
\hline \hline & 1995 & 2000 & 2005 & 2009 \\
\hline \hline France & 1,92 & 2,05 & 2,06 & 1,96 \\
Allemagne & 2,95 & 3,15 & 3,06 & 3,11 \\
Italie & 2,16 & 2,25 & 2,60 & 2,83 \\
Espagne & 0,82 & 0,91 & 1,30 & 1,02 \\
Portugal & 1,02 & 0,75 & 0,68 & 0,65 \\
UE 15 & 1,81 & 1,90 & 1,92 & 1,90 \\
UE 27 & 1,75 & 1,83 & 1,83 & 1,77 \\
Zone euro & 1,95 & 2,06 & 2,07 & 2,04 \\
\hline \hline
\end{tabular}

Note : l'intensité commerciale se définit comme le rapport entre la dépendance commerciale et la part des échanges commerciaux (exportations et importations) de la Suisse dans les échanges commerciaux au niveau mondial.

Source : calculs des auteurs à partir de données CHELEM (Cepii).

Ces tableaux confirment l'existence d'une «zone naturelle d'échange » européenne pour la Suisse, en particulier avec l'Allemagne depuis 2000. Ce constat reflète la position commerciale de la Suisse qui se situe, comme nous l'avons évoqué précédemment, « entre deux mondes ", c'est-à-dire à la fois intégrée aux économies européennes, mais aussi à celles du reste du monde, notamment des États-Unis et de plus en plus des pays asiatiques (Chine et Japon). Si le dynamisme de ces pays a bénéficié au commerce helvétique du fait de la spécialisation de la Suisse, il s'agit également des conséquences de la volonté économique du pays de diversifier ses échanges sur le plan géographique. Pour les autorités économiques et politiques de la Suisse, c'est donc une justification supplémentaire de la non appartenance à l'UE et à la zone euro. Malgré tout, les relations économiques que la Suisse entretient avec l'UE et la zone euro restent encore prépondérantes dans le total des échanges helvétiques, reflétant une dépendance asymétrique. Il en est de même d'un point de vue financier : la Suisse doit bénéficier des principales avancées en matière d'intégration réalisées au sein de l'UE, sans compromettre sa décision de ne pas adhérer. C'est doublement important pour la Suisse. En premier lieu, la forte spécialisation dans les domaines bancaire et financier, qui constitue une véritable "préférence nationale de structure », oblige le pays à être relié aux marchés financiers larges et liquides de l'UE, mais pas seulement : l'internationalisation de l'activité de nombreux agents économiques du pays, couplée à l'étroitesse relative des marchés financiers locaux, oblige le pays à entretenir des relations financières internationalisées, que ce soit à travers les transactions, les emprunts ou les placements à l'étranger en diverses devises (tableau 3). 
Tableau 3 : répartition géographique des avoirs et engagements financiers des banques domiciliées en Suisse en 2009

Avoirs financiers

Zone euro

Royaume-Uni

Etats-Unis

Japon

Russie

Total
(1)

(2)

215467

344838

380145

56553

3801

1403829
15,3

24,5

27,0

4,0

0,2

100

Engagements financiers

(3)

245822

330733

18,4

177906

24,7

13,3

17513

1,3

$11398 \quad 0,8$

1335980

Notes : (1) montant en millions de francs suisses ; (2) en \% du total des avoirs suisses à l'étranger ; (3) montant en millions de francs suisses ; (4) en \% du total des engagements suisses vis-à-vis de l'étranger.

Source : Banque nationale suisse (2009) et banques suisses $(2009,2010)$.

De ce fait, si la Suisse a l'obligation de participer à l'intégration financière en Europe, celle-ci ne doit pas être exclusive non plus, car la Suisse est aussi « entre deux mondes » d'un point de vue financier. En second lieu toutefois, le degré élevé d'intégration économique de facto de la Suisse à l'UE entraîne des effets d'engrenage au niveau de l'intégration financière: l'intégration économique, en supprimant les frontières nationales, facilite les échanges de biens, de services et des facteurs de production, notamment le capital qui transite par les marchés financiers au sens large. En retour, si l'intégration financière fonctionne selon une logique «propre », puisqu'un de ses objectifs est l'unification des activités financières au sein de la zone, elle vient aussi faciliter les transactions quotidiennes par l'intermédiaire d'un système de paiements unifié et unique. De la sorte, les paiements transitent progressivement par un seul et même système, maximisant les économies d'échelle, et interagissant également avec le développement réticulaire des échanges.

Ainsi, le fait que tout transite par un seul et même système dont les différents « nœuds » sont interconnectés et interdépendants peut être préjudiciable pour les pays tiers comme la Suisse. Celle-ci doit composer financièrement avec une zone qui fonctionne selon des règles propres, et où une dynamique interne discriminante risque de s'appliquer. Il est donc fondamental que la Suisse participe aux grandes avancées de l'intégration financière de l'UE, comme le Single Euro Payments Area (SEPA), qui correspond au système de paiement de masse unique au sein de la zone euro. La place financière suisse y est rattachée depuis 2006, puisque les banques et les associations bancaires suisses font partie de l'European Payments Council (EPC).

Or l'approfondissement de l'intégration économique, monétaire et financière au sein de l'UE depuis 1999 a renforcé cette dépendance extérieure de la Suisse, qui se matérialise en 
particulier par le fait que l'euro est devenu une devise centrale pour l'économie du pays. Ainsi, si un tel processus peut être source d'avantages pour la Suisse (simplification du commerce et des investissements avec l'UE, stabilité monétaire accrue en Europe), il représente également des contraintes. Désormais, ces risques de change sont concentrés, d'autant qu'en tant que monnaie de facturation des échanges, de comptabilité et de paiement, le franc suisse a vu son rôle international décliner au profit de l'euro ${ }^{6}$.

Si la position «d'intégration non adhésive » de la Suisse possède en apparence des avantages indéniables en matière d'autonomie décisionnelle, elle place simultanément le pays dans une situation fragile. De ce fait, la parité franc suisse/euro devient centrale pour le pays, ce qui semble alors fortement l'exposer aux variations de la monnaie nationale face à la devise européenne, et compromettre son autonomie monétaire, comme nous l'expliquons ci-après.

\subsection{Un degré élevé d'exposition aux variations de la parité franc suisse/euro?}

Compte tenu de son poids dans les échanges extérieurs de la Suisse, la zone euro occupe une place prépondérante dans le calcul du taux de change effectif nominal et réel de la Suisse réalisé par la BNS (environ 62\%). De ce fait, quand on aborde la question de l'exposition de l'économie helvétique aux variations du taux de change, la parité bilatérale franc suisse/euro est centrale. Or la plupart des études réalisées sur le degré d'exposition de l'économie helvétique aux variations du taux de change, par rapport à leur transmission ou à la volatilité notamment, et leurs conséquences supposées négatives sur l'économie suisse révèlent un impact non significatif des variations du taux de change effectif sur celle-ci. Tout d'abord, Stulz (2007) montre, à l'aide de modèles VAR identifiés de manière récursive, que la transmission des variations du taux de change à l'inflation s'est globalement atténuée depuis la décennie 1990 en Suisse. A partir du traitement de données mensuelles couvrant la période allant de janvier 1976 à décembre 2004, et en étudiant l'effet des variations du taux de change effectif nominal sur les prix à l'importation et à la consommation en Suisse, il parvient aux résultats suivants :

- les chocs de taux de change semblent se transmettre de façon importante aux prix à l'importation, bien que de façon non complète ;

\footnotetext{
${ }^{6}$ Voir, par exemple, Plihon et Guillaumin (2008).
} 
- une fois le choc survenu, la transmission des prix à l'importation en direction des prix à la consommation est une fois encore assez prononcée, du fait notamment de la part élevée des biens importés dans la consommation totale en Suisse ;

- enfin, la transmission d'un choc de taux de change aux prix à la consommation est beaucoup plus faible, les prix à la consommation ne réagissant que de façon marginale.

Dans une même optique, Fischer (1999) indique, à partir de données mensuelles couvrant la période de janvier 1973 à juin 1998, que la volatilité du franc suisse n'a exercé d'effets significatifs ni sur le volume des exportations, ni sur les prix de ces dernières. En utilisant un modèle empirique GARCH à variables multiples, il estime les rapports entre le commerce extérieur et la volatilité du taux de change du franc suisse, ainsi qu'entre la volatilité et les informations du passé, en partant de l'hypothèse que le risque de change est le seul risque. Cette constatation s'applique non seulement au total des exportations, mais également aux trois catégories d'exportations qu'il examine séparément, à savoir les produits semi-ouvrés, les biens de consommation et les biens d'équipement.

Pourtant, l'histoire monétaire contemporaine est marquée par de fréquentes variations du taux de change, qui sont potentiellement sources de perturbations pour l'économie nationale. En particulier, comme les pays européens constituent de longue date les principaux partenaires de la Suisse, les mouvements de la parité franc suisse/deutschemark puis franc suisse/euro sont les plus problématiques. 

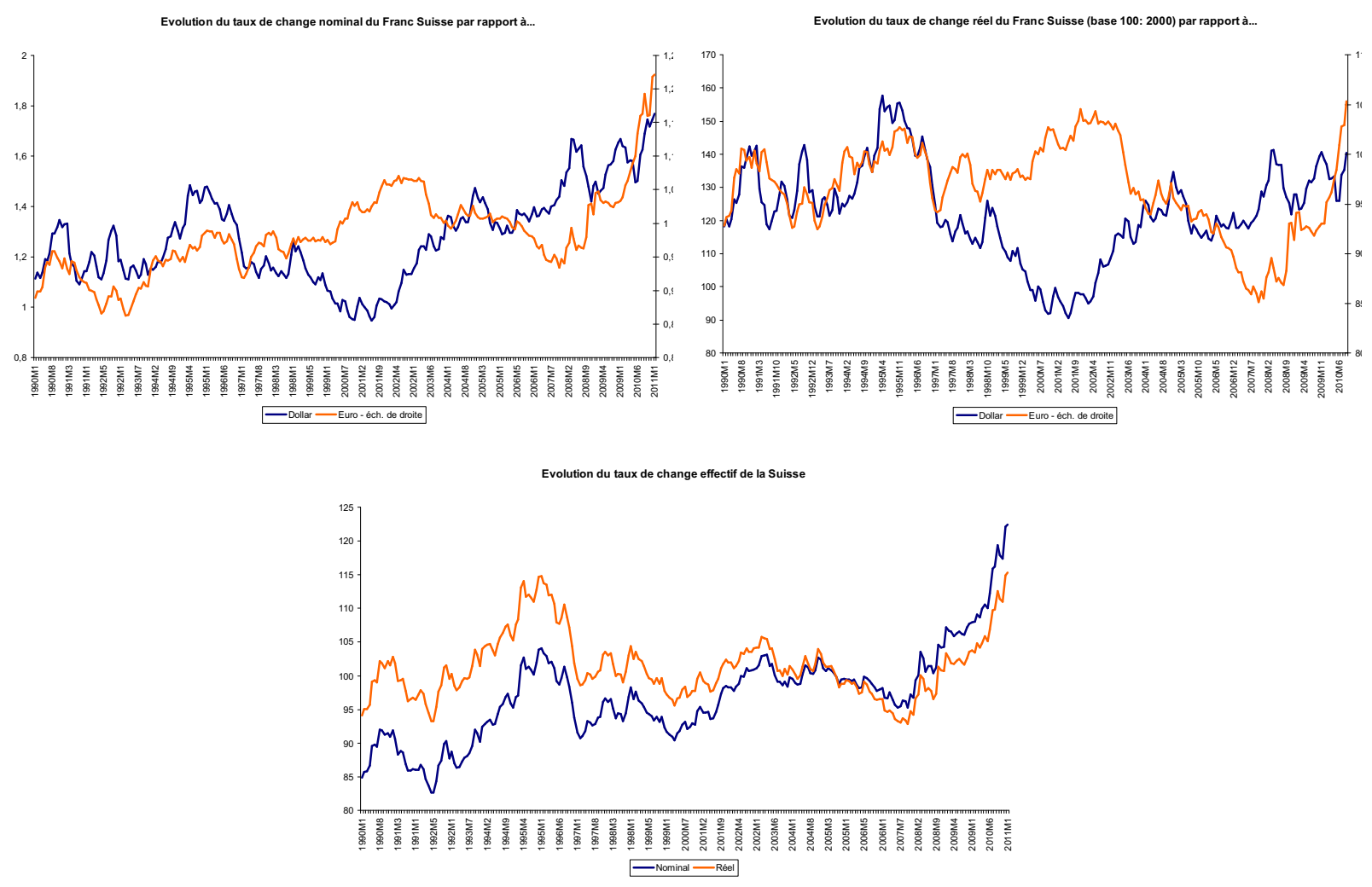

Graphique 2 : évolution du taux de change de la Suisse.

Source : Banque des règlements internationaux et $\mathrm{Fed}$ de Saint-Louis.

Entre février 2003 et janvier 2008, le franc suisse a connu une perte de sa valeur vis-à-vis de l'euro d'environ $14,5 \%$ en terme nominal. Avec la politique monétaire très accommodante mise en œuvre par la BNS à partir de mars 2003 où la bande de fluctuation assignée au taux d'intérêt de référence de la Banque centrale (le Libor à 3 mois en francs suisses) est $0-1 \%$, le franc suisse se déprécie vis-à-vis de l'euro, alors que le taux d'intérêt de la BCE est plus élevé. Malgré la normalisation de la politique monétaire de la part de la BNS débutée fin 2005, cette tendance se poursuit jusqu'à la fin du deuxième semestre de 2007.

Ce phénomène peut, en partie, être relié à la faiblesse relative du taux d'intérêt de la BNS, dont le niveau a été, durant cette période, inférieur à celui de la BCE. Autrement dit, le différentiel de taux d'intérêt favorable à la Suisse a incité les investisseurs internationaux à recourir à des emprunts importants en francs suisses pour investir dans des placements à plus forte rémunération, ou pour les prêter à nouveau à un taux plus élevé, en particulier dans la zone euro. Cette stratégie de «carry trade » a eu tendance à affaiblir le franc suisse face à l'euro sur le marché des changes, la devise helvétique jouant le rôle de monnaie de financement et la devise européenne celui de monnaie de placement (Galati et alii, 2007). Or si une monnaie de financement tend à se déprécier vis-à-vis d'une monnaie cible à court terme, elle s'expose surtout à des phénomènes de surréaction qui entraînent une appréciation 
brutale du taux de change (Frankel, 2007). Il semble que la Suisse ait connu ce scénario, face au dollar, mais surtout face à l'euro, puisque l'on observe une forte appréciation du taux de change face à l'euro essentiellement à partir de mars 2009.

Cette appréciation soudaine et significative du franc suisse face à l'euro s'explique aussi par le rôle de valeur refuge internationale qu'il exerce : avec les turbulences récentes au sein de la zone euro, les investisseurs internationaux ont déplacé leurs capitaux en Suisse, dont les caractéristiques les rassurent (stabilité monétaire, fiscale et politique, secret bancaire, marchés financiers liquides, performance du secteur bancaire,...). Ce phénomène s'était déjà observé après la crise financière de 1987, lors de la crise du Système monétaire européen en 1992 et dans une moindre mesure après les attentats de 2001.

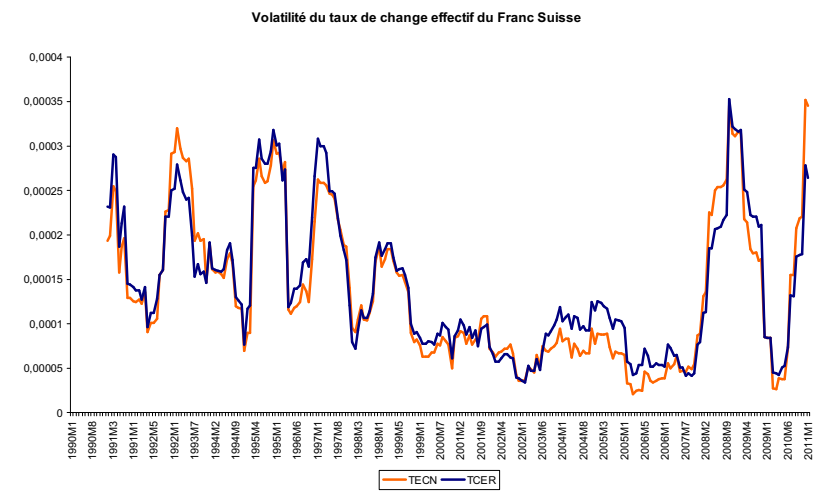

Graphique 3 : volatilité du taux de change de la Suisse.

Source : calculs des auteurs à partir de données de la Banque des règlements internationaux.

Or quand le franc suisse est considéré comme une valeur refuge, le pays enregistre des entrées massives de capitaux qui correspondent à une demande soudaine d'actifs libellés en francs suisses, et qui, compte tenu de l'étroitesse relative des marchés financiers helvétiques, se traduisent par une appréciation brutale du taux de change sans rapport avec les fondamentaux économiques, mais simplement du fait du rôle international particulier du franc suisse.

Pour Ranaldo et Söderlind (2007) en effet, le franc suisse possède toutes les caractéristiques et tous les signes d'une monnaie refuge : outre la stabilité politique et le secret bancaire évoqués jusqu'ici, les auteurs rappellent les atouts de la monnaie helvétique, qui induit la sécurité et la valorisation des actifs libellés en francs suisses. La tradition historique de lutte contre l'inflation de la Banque centrale du pays, couplée à la tendance haussière du franc suisse sur les marchés des changes, créent en effet les conditions d'une faible dépréciation, voire d'une valorisation du capital. De plus, l'internationalisation financière, et la qualité de l'offre financière et bancaire nationales, rendent particulièrement attractifs les investissements de court terme en Suisse. L'offre semble très élastique en cas de turbulences de marché. Leur 
étude indique dans ce cadre que lorsque des perturbations majeures surviennent, le cours du franc suisse évolue inversement aux rendements de marché et de la perception des risques internationaux. En particulier, la baisse des principales valeurs boursières sur les marchés internationaux est associée, d'après leurs observations, à une appréciation du franc suisse sur le marché des changes. Leur étude indique donc que la valeur du franc suisse varie de façon inverse à celle de certains actifs financiers internationaux révélateurs de tendance, mais aussi à certains événements non économiques, bancaires ou financiers.

Or comme évoqué supra, le problème, à partir de là, est que l'appréciation de la parité franc suisse/euro semble constituer une réelle contrainte structurelle pour certains agents économiques en Suisse (les banques, les industries et les PME exportatrices) mais surtout pour la BNS, susceptible de connaître des difficultés à assurer ses engagements intérieurs à cause de ces perturbations extérieures. Même si la BNS n'est pas une banque commerciale qui surveille ses profits ${ }^{7}$, cette augmentation implique malgré tout des risques de change significativement élevés, donc des pertes comptables sur les positions de change, ainsi que l'augmentation de la base monétaire. Pour donner un ordre d'idées, la BNS a subi une perte de 3 milliards de francs suisses en mars 2010 et une autre de 8,5 milliards en juin de la même année du fait de ses interventions : en proportion du PIB, cela équivaudrait pour la Fed à une perte de 200 milliards de dollars. Mais plus largement, cela oblige la BNS à repenser ses instruments pour la conduite de la politique monétaire du fait de la quantité de liquidités en circulation, qui s'apparente à un «hyperpragmatisme périlleux » et à une " liberté monétaire contrainte ». Cette dernière pose donc la question de son indépendance et de son autonomie réelles à l'égard de l'UE, et plus largement celle de la viabilité de la situation d' « intégration non adhésive » helvétique.

Dans cette perspective, compte tenu de la volonté politique suisse de ne pas adhérer à l'UE qui mettrait pourtant fin, à terme, aux fluctuations nominales de la parité franc suisse/euro, un « arrangement monétaire médian » du type ancrage de jure peut être envisagé.

\footnotetext{
${ }^{7}$ Bien que les cantons suisses, qui sont actionnaires de la BNS, sont soucieux de ces profits puisqu'ils en tirent des ressources. Or l'utilisation des réserves de change pour les interventions sur le marché des changes a conduit à une érosion de ceux-ci, d'où les critiques des cantons à l'égard de la politique monétaire menée par la BNS.
} 


\section{L'ancrage officiel du franc suisse à l'euro: une dépendance monétaire libératrice?}

Le franc suisse semblant régulièrement ancré de facto à l'euro, la question de l'officialisation d'un tel régime de change se pose. C'est pourquoi il convient de passer en revue les coûts et les bénéfices potentiels de celui-ci pour la Suisse.

\subsection{Le franc suisse, un « étrange animal » ancré de facto à l'euro}

Si le discours officiel des autorités monétaires helvétiques est d'afficher l'autonomie monétaire du pays vis-à-vis de l'intégration monétaire européenne, la réalité semble différente. Au regard de l'histoire monétaire contemporaine de la Suisse, le franc suisse n'est pas entièrement caractérisé par un régime de taux de change flottant «pur » vis-à-vis des principales devises internationales (Reinhart et Rogoff, 2004). Les résultats de leur recherche indiquent que la Banque nationale suisse n'a pas suivi dans les faits le régime de change annoncé : elle a souvent établi ou bénéficié d'un ancrage officieux de la monnaie nationale aux devises de ses principaux partenaires. Si dans le système de Bretton Woods, le franc suisse est ancré officiellement au dollar, la période du flottement du taux de change qui suit est marquée par un "flottement contrôlé » du franc suisse, voire un ancrage de facto du franc suisse au deutschemark puis à l'euro. De façon intéressante, les auteurs montrent également que l'ancrage aux devises européennes s'est effectué dans une bande très étroite de $+/-2 \%$. Le renforcement de l'intégration économique et monétaire européenne n'a pas modifié le comportement du franc suisse sur le marché des changes, puisque la bande étroite de fluctuation du franc suisse face à l'euro est la même que face au deutschemark (tableau 4).

Tableau 4 : régimes de change de facto de la Suisse $(1949-2007)$

\begin{tabular}{lcc}
\hline \hline & Régime de change & Commentaires \\
\hline \hline $1949-1973$ & Ancrage au dollar & Contrôle des capitaux \\
$1973-1981$ & Flottement contrôlé & - \\
$1981-1998$ & Bande flottante vis-à-vis du deutsche mark & $+/-2 \%$ \\
$1999-2007$ & Bande flottante vis-à-vis de l'euro & $+/-2 \%$ \\
\hline \hline
\end{tabular}

Source : Reinhart et Rogoff (2004).

Ces conclusions paraissent surprenantes, puisque la Suisse n'a plus d'objectif de change explicite depuis 1980 : après l'ancrage du franc suisse au deutschemark de 1978 à 1979, la BNS a renoncé à poursuivre officiellement une telle stratégie. Le franc suisse aurait donc dû 
fluctuer davantage face aux deux devises européennes. C'est même a priori paradoxal dans un système de taux de change flottants, où le cours des monnaies suit théoriquement une marche aléatoire et avec des mouvements inattendus (Obstfeld et Rogoff, 1995). C'est d'autant plus le cas pour le franc suisse : compte tenu de son rôle international évoqué précédemment, cette devise est fréquemment échangée sur le marché des changes, ce qui devrait avoir une incidence sur la variabilité et la volatilité de ses différentes parités. Or ce n'est pas le cas, en particulier au cours de la dernière décennie où la monnaie nationale helvétique a été ancrée de facto à l'euro. De ce fait, puisqu'un tel régime de change semble en apparence comporter des désavantages pour la Suisse, notamment parce que la fonction de valeur refuge internationale jouée par le franc suisse induit une appréciation de la parité franc suisse/euro qui nécessite d'être combattue par de fréquentes et massives interventions de la BNS sur le marché des changes, la Suisse pourrait envisager un ancrage de jure du franc suisse à l'euro. Cette option est pourtant balayée par la BNS, qui met en avant les désavantages d'un tel régime de change, surpassant ses bénéfices.

\subsection{Les coûts potentiels de l'ancrage de jure}

Tout d'abord, le premier coût potentiel vient de la nature nécessairement «asymétrique » de l'ancrage officiel du franc suisse à l'euro sur le plan institutionnel. En effet, la stabilité nominale du taux de change recherchée via l'ancrage officiel d'une monnaie à l'euro renvoie à un des critères essentiels de pré adhésion du Traité de Maastricht. Cela signifie que dans l'esprit et les principes de l'intégration européenne, il est nécessaire d'adhérer à l'UE en premier lieu avant d'envisager une telle perspective. Comme la Suisse ne souhaite pas l'adhésion, cela l'exclut de fait.

En supposant que l'ancrage officiel atteigne son principal objectif, à savoir parvenir à la stabilisation nominale du taux de change franc suisse/euro, il n'est pas certain que cette «stabilité organisée » aboutisse à une absence de fluctuation du taux de change réel. Une politique d'ancrage officiel à l'euro serait également le signal public que l'institut monétaire suisse accorde de l'importance uniquement à la parité entre le franc suisse et l'euro, au détriment d'autres cours bilatéraux. Mais le principal coût de l'ancrage officiel le plus mis en avant est qu'il implique de fait une perte de souveraineté de la politique monétaire, dont le degré dépend de l'importance de la parité ciblée dans le taux de change effectif du pays, et de l'ouverture économique du pays vis-à-vis de la zone considérée. Comme ces deux dimensions sont fortement présentes pour la Suisse, ceci entraînerait une perte d'autonomie 
proportionnelle qui risquerait d'avoir une conséquence fortement préjudiciable: les conditions monétaires de la Suisse se calqueraient fortement sur celles de la zone euro avec le risque cette fois que les deux monnaies ne soient plus perçues comme distinguables, et que les taux d'intérêt helvétiques se mettent au niveau de ceux en vigueur dans la zone euro. Cette perspective pourrait être particulièrement coûteuse pour la Suisse puisque, historiquement, le pays jouit de taux d'intérêt réels à court terme et à long terme très faibles, et inférieurs à ceux des principaux partenaires, situation qualifiée de «bonus » de taux d'intérêt. Cela crée, toutes choses égales par ailleurs, un environnement favorable pour l'emprunt, l'investissement et la croissance.

De même, la place financière suisse bénéficie de ce «bonus», en lien avec le rôle international $\mathrm{du}$ franc suisse en tant que valeur refuge ou monnaie de diversification des placements. Les investisseurs internationaux se dirigent vers la Suisse pour diversifier leurs placements ou se protéger d'un certain nombre de risques (UBS, 2000). D'où une hausse de la valeur des actifs libellés en francs suisses, compte tenu de la taille relative des marchés financiers suisses.

Le franc suisse est recherché parce que ses fluctuations sont perçues comme peu corrélées avec celles des autres. Or si le franc suisse se situe dans une bande étroite face à l'euro suite à l'ancrage, la volatilité est faible et la corrélation entre le franc suisse et l'euro est forte. De ce fait, le franc suisse ne joue plus son rôle d'actif international indépendant, les actifs libellés dans cette monnaie sont moins demandés, la place financière helvétique perd de son attractivité et le «bonus » se réduit ou disparaît. Comme le «bonus » est indispensable à la compétitivité de la Suisse, il est nécessaire d'éviter l'ancrage officiel du franc suisse à l'euro. L'étude de la parité franc suisse/euro montre que l'existence d'une bande de fluctuations étroite entre les deux monnaies se traduit par un effacement des particularités du franc suisse, et donc par une diminution du différentiel de taux d'intérêt. De ce fait, toute l'économie suisse serait pénalisée par cette remontée des taux.

Les obstacles à l'ancrage officiel du franc suisse à l'euro semblent donc nombreux, comme l'illustre le cas de la perte redoutée partielle ou totale du «bonus » en cas d'adoption de ce régime de change. Les autorités monétaires suisses mettent même en avant que malgré ces contraintes « objectives », la BNS parvient à assurer sa mission de stabilité des prix. Pour la BNS, le degré élevé d'intégration économique et monétaire à la zone euro et une corrélation élevée des chocs conduisent nécessairement à des réponses similaires de politiques monétaires qui ne doivent pas être interprétés comme une «vassalisation » monétaire. Au contraire, dans une petite économie ouverte comme la Suisse, il est normal que l'économie 
soit exposée aux chocs extérieurs, et notamment aux variations du taux de change. Dans cette perspective, répondre aux fluctuations de la parité franc suisse/euro s'apparente à une politique monétaire optimale qui ne doit pas être confondue avec une hétéronomie monétaire. C'est pour cette raison que l'ancrage officiel est aussi repoussé, même s'il présente aussi des avantages susceptibles de contrebalancer ses coûts potentiels. Nous les présentons ci-après.

\subsection{Les bénéfices potentiels de l'ancrage de jure}

Genberg (2000) explique que dans le cas suisse, vouloir poser le débat entre flottement pur du taux de change franc suisse/euro et union monétaire avec la zone euro est réducteur. En d'autres termes, un flottement administré prenant la forme d'un ancrage officiel à l'euro rigide ou souple - pourrait être préférable à ces deux solutions extrêmes. Son argumentation s'appuie sur la présence d'avantages indéniables pour le pays, que nous abordons ici.

Tout d'abord, contrairement à la position défendue officiellement par les autorités monétaires helvétiques, il semble qu'une telle modalité d'intégration monétaire "médiane » à la zone euro convienne aux particularités de la Suisse, compte tenu de son exposition aux chocs en provenance de la zone euro. Le premier avantage indéniable pour l'économie suisse de l'ancrage de jure serait qu'il stabiliserait la parité nominale franc suisse/euro, et donc que les agents économiques helvétiques pourraient prendre leurs décisions dans un univers moins incertain. Une petite économie ouverte comme la Suisse doit en effet se prémunir des chocs de taux de change, du fait de l'internationalisation de ses activités. En particulier, une parité stabilisée améliorerait l'horizon prévisionnel des entreprises exportatrices helvétiques : les PME sont fortement pénalisées par des variations erratiques du taux de change franc suisse/euro, notamment dans le sens appréciatif comme nous l'avons indiqué. Elles ont, qui plus est, davantage de difficultés à se couvrir en matière de risques de change associés, et doivent par conséquent faire preuve de flexibilité organisationnelle qui n'est pas aisée à mettre en œuvre dans les faits.

Si l'ancrage officiel n'est pas l'adhésion intégrale, des effets intégrateurs «endogènes » du type de ceux mis en évidence par Frankel et Rose $(1997,1998)$ pourraient survenir. Or des échanges plus intenses se traduiraient par une convergence plus importante des cycles économiques, ce qui rendrait plus soutenable ex post la parité ciblée et l'ancrage officiel. Grâce à cet environnement stabilisé, la BNS pourrait alors se concentrer prioritairement sur des considérations internes, donc disposer là aussi de plus d'autonomie réelle. 
Il découle du point précédent un autre avantage de l'ancrage officiel. Selon l'ampleur des chocs économique et monétaire en provenance de la zone euro affectant ce petit pays, il n'est pas certain qu'une politique monétaire isolée parvienne à les résorber. L'échec des interventions récentes de la BNS pour tenter d'inverser la tendance appréciative de la monnaie nationale face à l'euro le rappelle parfaitement. L'insularité institutionnelle de la politique monétaire suisse dans le contexte politique d'un refus d'adhésion totale à l'UE pose ainsi la question de son degré d'indépendance et d'autonomie effective à l'égard de la zone euro. Or la dépendance économique et financière de la Suisse à l'égard de la zone euro apparaît trop forte pour ne pas entrer en contradiction avec le flottement libre de la monnaie nationale.

En tenant compte de la situation helvétique, il est certain que celui-ci ne peut véritablement exister dans les faits, obligeant la plupart du temps la Banque centrale à définir une ou plusieurs cibles implicites du taux de change comme nous l'avons mis en évidence supra, donc à être prête à intervenir de façon répétée sur le marché des changes, avec tous les risques qu'une telle politique implique. En particulier, en renversant un des arguments en défaveur de l'ancrage officiel, ce régime de change peut au contraire s'avérer plus efficace que le simple ancrage de facto pour éviter les attaques spéculatives : compte tenu du potentiel appréciatif du franc suisse face à l'euro et des interventions répétées de la BNS pour contrecarrer cette tendance en s'accrochant au libre flottement officiel, des spéculateurs pourraient vouloir tester la soutenabilité d'une telle politique.

Malgré les éléments positifs précédents, la principale interrogation liée à la perspective de l'ancrage officiel demeure l'avenir du «bonus », c'est-à-dire qu'il pose la question de la possibilité pour la Suisse de préserver celui-ci. Il risque de disparaître ou de diminuer du fait des implications de l'ancrage officiel, notamment pour les taux d'intérêt de long terme. Par contre, si celui-ci trouve ses origines dans des facteurs structurels propres à l'économie suisse, il n'y a pas de raison pour qu'il ne perdure pas : le cas actuel de la zone euro montre que les investisseurs continuent de distinguer des particularités propres à chaque pays, conditionnant la prime liée au risque souverain qu'ils demandent. Si une certaine convergence s'opère du fait de l'intégration monétaire, les écarts ne sont donc pas complètement supprimés (Sfreddo, 2002).

Par ailleurs, si les régimes de changes flottants semblent avoir tout de même un avantage par rapport aux régimes de changes fixes concernant l'ajustement aux chocs macroéconomiques, il faut cependant différencier l'origine du choc (nominale ou réelle). Or d'un point de vue théorique, les changes fixes sont mieux à même de protéger les économies contre les chocs nominaux alors que les changes flottants seraient plus appropriés en cas de chocs réels (Calvo 
et Mishkin, 2003). Confronté à un choc nominal, un taux de change fixe joue un rôle stabilisateur puisqu'il impose une discipline monétaire ; à l'opposé, un taux de change flottant aurait tendance à transmettre la perturbation à la sphère réelle (Clarida et Gali, 1994), augmentant ainsi la volatilité de l'économie. En cas de choc réel, sur les termes de l'échange par exemple, les changes flottants apportent une meilleure réponse. Edwards et Levy-Yeyati (2003) confirment ces conclusions théoriques mais leurs résultats apparaissent "isolés" dans la littérature économique même s'ils confirment, comme Levy-Yeyati et Sturzenegger (2003), que les pays ayant opté pour un change flottant obtiennent une croissance plus rapide et plus élevée que ceux qui ont opté pour un change fixe ${ }^{8}$. Cependant, il apparaît que les taux de change flottants ne parviennent pas totalement à atténuer les chocs et auraient même tendance à les amplifier (Frankel et Rose, 1994 ; Hausmann et alii, 1999).

C'est pourquoi, pour déterminer l'opportunité et la viabilité d'un ancrage de jure du franc suisse à l'euro, il convient de mettre en évidence la nature des chocs auxquels est soumise la Suisse : si son économie est affectée essentiellement par des chocs nominaux, alors il existe un intérêt pour la mise en place d'un taux de change fixe ; à l'inverse, si le pays est touché majoritairement par des chocs réels, le flottement du taux de change doit être préservé. Nous tentons par conséquent d'apporter une réponse à cette question dans la partie suivante.

\section{Les sources des fluctuations du taux de change suisse}

\subsection{La méthode SVAR}

Chaque variables macroéconomique peut être décomposée en deux composantes stochastiques : une permanente et une cyclique (ou temporaire). Il existe plusieurs méthodes pour effectuer cette décomposition. Nous choisissons d'adopter une méthodologie VAR structurel (SVAR par la suite) basée sur les travaux de Blanchard et Quah (1989) qui permet d'orthogonaliser les chocs en les décomposant selon leurs effets à long terme. Une décomposition employée précédemment était la décomposition de Cholesky qui ne fait appel à aucune théorie économique. L'inconvénient est que les résultats obtenus dépendent de l'ordre dans lequel sont rangées les séries «la matrice obtenue est triangulaire inférieure ; les variables sont rangées de la plus exogène à la plus endogène » (Lardic et Mignon, 2002). Les

\footnotetext{
${ }^{8}$ Nous pouvons également citer les travaux de Gosh et alii (1997) qui effectuent une synthèse sur l'efficacité des régimes de change. D'après leurs conclusions, les régimes de change fixe, dans le passé, ont été associés à un taux d'inflation plus faible et un investissement plus important mais également à une croissance plus faible. En règle générale, le régime de change fixe est choisi pour maitriser l'inflation qui impose une discipline monétaire. Mais, à la différence de Levy-Yeyati et Sturzenegger (2003), les conclusions de Gosh et alii (1997) montrent que, outre une inflation plus forte, les performances en terme de croissance sont médiocres.
} 
travaux de Blanchard et Quah (1989) corrigent cette « anomalie » en imposant des contraintes liées à la théorie économique, ce qui permet d'interpréter chaque choc comme choc d'offre, de demande, de politique monétaire....

Nous estimons un VAR sous forme réduite :

$$
A(L) X_{t}=u_{t}
$$

Avec $X_{t}=\left(\Delta r_{t}, \Delta e_{t}\right)^{\prime} . r$ est le logarithme du taux de change réel et $e$ le logarithme du taux de change nominal, $\Delta$ indique les variations premières de ces variables et $u_{t}$ est un bruit blanc, de moyenne nulle et de variance $\sigma^{2}$.

La forme moyenne mobile infinie (VMA) du processus s'écrit :

$$
X_{t}=B(L) u_{t}
$$

On peut réécrire les erreurs de la forme réduite comme une combinaison linéaire, telle que : $u_{t}=S \varepsilon_{t}$ avec $\varepsilon_{t}$ les chocs structurels. Nous considérons ainsi un vecteur d'innovations normalisées et orthogonales $\varepsilon_{t}=\left(\varepsilon_{t}^{r}, \varepsilon_{t}^{n}\right)^{\prime}$, où $\varepsilon_{t}^{r}$ et $\varepsilon_{t}^{n}$ sont, respectivement, les chocs réel et nominal satisfaisants : $u_{t}=S \varepsilon_{t}$ et $E\left(\varepsilon \varepsilon^{\prime}\right)=I$. Ce qui implique que $S S^{\prime}=\Sigma$ et que la connaissance d'une matrice d'orthogonalisation $S$ permet d'écrire la représentation VMA en terme de chocs indépendants, dits structurels :

$$
X_{t}=C(L) \varepsilon_{t}
$$

Avec

$$
C(L)=B(L) S
$$

La forme développée de la matrice $C(L)$ s'écrit :

$$
C(L)=\sum_{i=0}^{\infty}\left[\begin{array}{ll}
c_{11, i} & c_{12, i} \\
c_{21, i} & c_{22, i}
\end{array}\right]
$$

La comparaison du nombre de paramètres à déterminer pour l'identification de la forme structurelle avec le nombre de paramètres effectivement estimés dans le modèle VAR montre que l'identification de la forme structurelle nécessite l'imposition de $n(n-1) / 2$ contraintes. Nous avons ainsi besoin de 1 contrainte. Grâce aux travaux de Blanchard et Quah (1989) et Clarida et Gali (1994), nous imposons la restriction suivante : un choc nominal sur le niveau du taux de change réel est nul à long terme, soit $\sum_{i=0}^{\infty} c_{12, i}=0$. 


\subsection{Les données}

La méthode décrite dans la section 4.1 est appliquée aux taux de change effectifs ainsi qu'aux taux de change bilatéraux de la Suisse. Les taux de change bilatéraux de la Suisse sont exprimés vis-à-vis du dollar américain. Les taux de change effectifs proviennent de la Banque des règlements internationaux. Il s'agit de données mensuelles basées sur le commerce avec 27 pays. Les données pour les taux de change bilatéraux nominaux proviennent de la FED de Saint-Louis. Les données des indices de prix à la consommation sont issues des Statistiques Financières Internationales du Fonds monétaire international ${ }^{9}$. Nous construisons le taux de change réel bilatéral de la Suisse à l'aide de l'indice des prix à la consommation. Les taux de change nominaux et les indices de prix à la consommation sont préalablement transformés dans la même base (2000).

L'ensemble des données utilisées sont mensuelles, sur la période 1990.1 à 2010.12. Toutes les variables ont été transformées en logarithme. Les variables sont soumises à des tests de racines unitaires. Les variables sont stationnaires en différence première. L'étude de la cointégration des variables ne révèle pas de relation de long terme entre ces dernières. Enfin, en utilisant les critères d'information d'Akaike, de Schwartz et d'Hannan-Quinn ${ }^{10}$, nous spécifions un nombre de retards égal à, respectivement, 2 et 4 pour les modèles de taux de change bilatéraux et de taux de change effectifs ${ }^{11}$.

\subsection{Les résultats}

L'analyse des résultats se focalisera sur deux points essentiels : la décomposition de la variance et les fonctions de réponses aux chocs.

\section{La décomposition de la variance}

La décomposition de la variance nous permet d'évaluer, à différents horizons temporels, l'importance des différents chocs (d'offre et de demande ou réel et nominal) sur les fluctuations des variables du modèle.

Les tableaux 5 et 6 présentent les résultats de cette décomposition. Comme nous imposons qu'un choc nominal n'a aucun effet à long terme sur le taux de change réel, la proportion de la

\footnotetext{
${ }^{9}$ Ligne ae.

${ }^{10}$ Ces critères sont les plus utilisés mais il en existe d'autres (Deniau et alii, 1992).

${ }^{11}$ Le détail des tests de racine unitaire et de cointégration ainsi que le détail des critères d'information sont disponibles auprès des auteurs sur simple demande.
} 
variance du taux de change réel expliquée par les chocs réels tend, graduellement, vers 100\%, au fur et à mesure que l'horizon augmente.

L'analyse du taux de change effectif réel montre que les chocs réels sont, de loin, la source la plus importante de fluctuations du taux de change effectif réel. Nous observons que l'importance relative des chocs nominaux dans les fluctuations du taux de change effectif réel ne cesse de diminuer avec l'horizon temporel ets représente moins de $1 \%$ de la variance expliquée.

Tableau 5 : décomposition de la variance du taux de change effectif

Taux de change effectif réel

\begin{tabular}{ccc}
\hline \hline Horizon & Contribution relative des chocs réels & Contribution relative des chocs nominaux \\
\hline 1 & 94,2 & 5,8 \\
2 & 94,8 & 5,2 \\
3 & 95,2 & 4,8 \\
4 & 96,0 & 4,0 \\
8 & 97,7 & 2,3 \\
12 & 98,4 & 1,6 \\
40 & 99,5 & 0,5 \\
\hline & Taux de change effectif nominal \\
\hline & 95,3 & 4,7 \\
2 & 90,4 & 9,6 \\
3 & 89,4 & 10,6 \\
4 & 87,2 & 12,8 \\
8 & 85,5 & 14,5 \\
12 & 85,2 & 14,8 \\
40 & 84,7 & 15,3 \\
\hline \hline
\end{tabular}

L'analyse du taux de change réel bilatéral nous montre que les fluctuations attribuables aux chocs réels sont plus importantes que celles causées par les chocs nominaux. Au mieux, à peine $8 \%$ des chocs nominaux expliquent la variance du taux de change effectif réel. Pour l'analyse du taux de change effectif nominal, nous observons que les chocs nominaux expliquent une très faible part de la variance du taux de change effectif nominal. Ces derniers sont davantage expliqués par les chocs réels.

Ces résultats sont alors compatibles avec l'idée selon laquelle, sous un régime de changes fixes, la présence de chocs nominaux touchant le taux de change réel est faible. 
Tableau 6 : décomposition de la variance du taux de change bilatéral

Taux de change réel

\begin{tabular}{ccc}
\hline \hline Horizon & Contribution relative des chocs réels & Contribution relative des chocs nominaux \\
\hline 1 & 98,2 & 1,8 \\
2 & 98,6 & 1,4 \\
3 & 99,0 & 1,0 \\
4 & 99,2 & 0,8 \\
8 & 99,6 & 0,4 \\
12 & 99,7 & 0,3 \\
40 & 99,9 & 0,1 \\
\hline & & Taux de change nominal \\
\hline & 95,7 & 4,3 \\
2 & 95,3 & 4,7 \\
3 & 94,3 & 5,7 \\
4 & 94,2 & 5,8 \\
8 & 94,1 & 5,9 \\
12 & 94,1 & 5,9 \\
40 & 94,1 & 5,9 \\
\hline \hline
\end{tabular}

Les fonctions de réponses aux chocs

Tableau 7 : réponse du taux de change effectif réel à un choc...

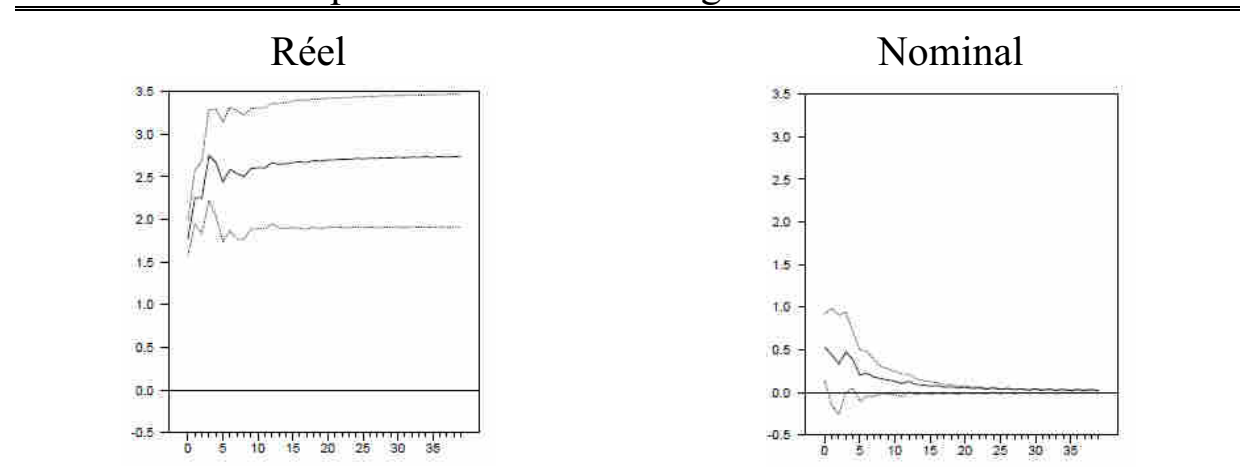

Tableau 7 bis : réponse du taux de change effectif nominal à un choc...

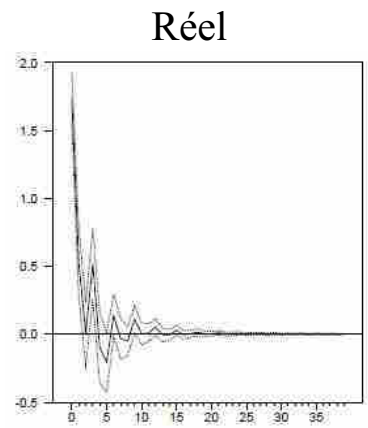

Nominal

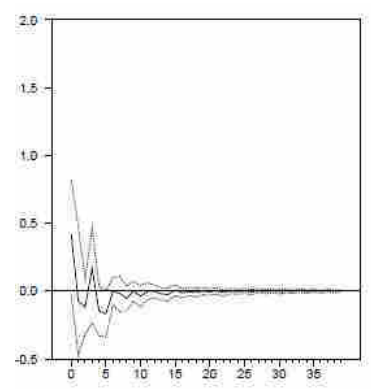


Tableau $8:$ réponse du taux de change bilatéral réel à un choc...
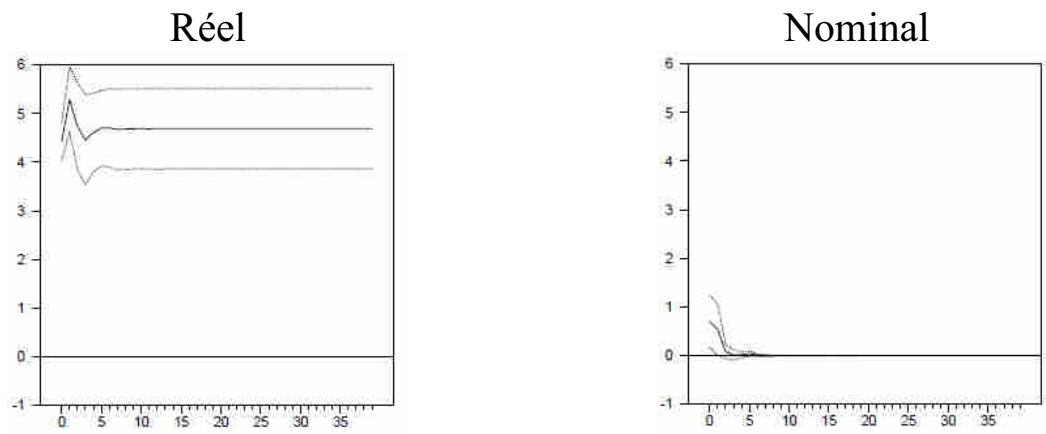

Tableau 8 bis : réponse du taux de change bilatéral nominal à un choc...
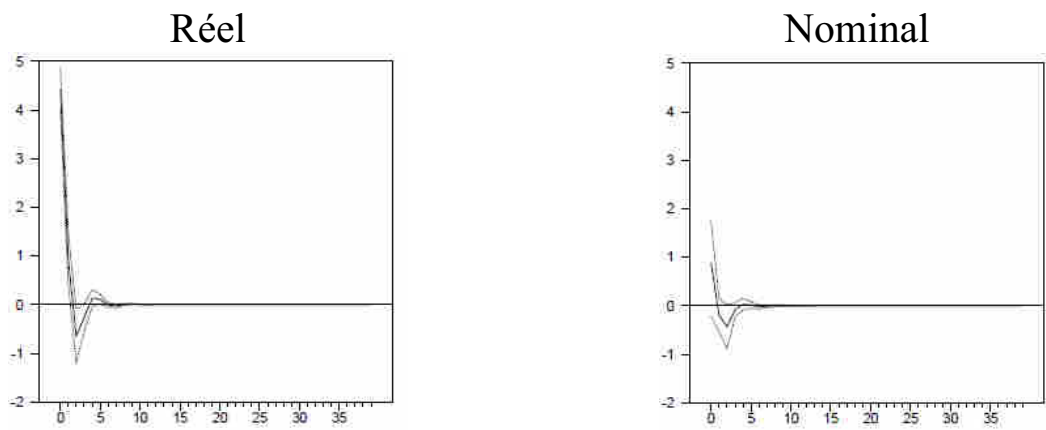

L'effet, à long terme, des chocs réels est toujours positif. La durée d'ajustement du taux de change réel bilatéral est plus rapide que celle du taux de change effectif réel. Nous constatons que la durée d'ajustement, quel que soit le taux de change étudié, à un choc structurel, réel ou nominal, est relativement rapide et ne dépasse pas 3 ans. Elle est même inférieure à 1 an dans la plupart des cas.

Au vu de l'ensemble des résultats, la Suisse pourrait dès lors s'ancrer à l'euro mais perdrait, en partie, son autonomie monétaire.

\section{Conclusion}

La posture politique de la Suisse à l'égard de l'UE s'apparente dans ce pays à un «psychodrame » permanent, au sens où les Suisses s'interrogent depuis de nombreuses années quant à la politique européenne «adéquate » à adopter. Cet article a tenté d'en rendre compte sous un aspect souvent peu mis en avant, c'est-à-dire monétaire, et plus précisément à travers la perspective d'un ancrage officiel du franc suisse à l'euro. En effet, si la Suisse est non membre de l'UE et bien qu'elle possède une orientation internationale de ses échanges, son économie reste malgré tout fortement dépendante des échanges économiques et financiers avec l'UE et plus particulièrement avec la zone euro. Or cette dépendance élevée se traduit par une forte exposition de la Suisse aux variations du taux de change franc suisse/euro, avec 
des répercussions négatives pour de nombreux agents économiques. Cette contrainte monétaire extérieure est renforcée par le fait que le franc suisse joue un rôle de valeur refuge internationale, avec comme conséquence une appréciation soudaine et massive face aux principales devises internationales, dont l'euro. Les récentes turbulences au sein de la zone euro ont ainsi provoqué un tel phénomène, qui a obligé les autorités monétaires helvétiques à réagir en intervenant sur le marché des changes, mais avec une faible efficacité et à un coût élevé.

C'est pourquoi, compte tenu de la volonté politique helvétique de ne pas adhérer à l'UE et à la zone euro, qui serait pourtant une solution pour mettre fin à ces variations nominales de la parité franc suisse/euro, nous avons cherché à savoir dans cet article si un ancrage officiel du franc suisse à l'euro pourrait constituer une alternative crédible et viable pour la Suisse. Nos tests économétriques montrent que ce serait le cas, mais avec une contrepartie importante, à savoir une diminution significative de l'autonomie monétaire du pays.

En conséquence, comme la Suisse et sa Banque centrale ont une solide réputation de crédibilité monétaire, il conviendrait de poursuivre les investigations quant au degré et aux implications de la diminution de l'autonomie monétaire du pays. En particulier, de futures études devraient chercher à quantifier les effets d'un tel ancrage officiel sur la perte de bienêtre suisse, et en particulier sur le «bonus » suisse de taux d'intérêt, sous différents aspects « techniques » d'un tel régime de change, c'est-à-dire ancrage souple ou rigide, avec des bandes de fluctuations ajustables ou pas.

\section{Bibliographie}

BANQUE NATIONALE SUISSE, 2009, « Comptes financiers suisses ».

BLANCHARD, O. et D. QUAH, 1989, “The Dynamics Effects of Aggregate Demand and Supply Disturbances", American Economic Review, vol. 79 (4), pp. 655-673.

CALVO, G. et F. MISHKIN, 2003, “The mirage of exchange rate regimes for emerging countries", NBER working paper 9808.

CLARIDA, R. et J. GALÍ, 1994, "Sources of Real Exchange Rate Fluctuations: How Important are Nominal Shocks?", NBER working paper 4658.

DENIAU, G., FIORI, G. et A. MATHIS, 1992, "Sélection du nombre de retards dans un modèle VAR, conséquences éventuelles du choix des critères", Economie et Prévision, 106, pp. 61-69. 
EDWARDS, S. et E. LEVY-YEYATI, 2003, "Flexible Exchange Rates as Shocks Absorbers", NBER working paper 9867.

FISHER, A., 1999, "Les effets de la volatilité des changes sur le commerce extérieur de la Suisse : estimations à partir d'un modèle à variables multiples", Bulletin trimestriel de la BNS, 2 .

FRANKEL, J., 2007, "Getting Carried Away: How the Carry Trade and Its Potential Unwinding Can Explain Movements in International Financial Markets", Milken Institute Review, November $19^{\text {th }}$.

FRANKEL, J. et A. ROSE, 1994, "A survey of empirical research on nominal exchange rates", NBER working paper 4865.

FRANKEL, J. et A. ROSE, 1997, “Is EMU more justifiable ex post than ex ante ?”, European Economic Review, vol. 41 (3-5), pp. 753-760.

FRANKEL, J. et A. ROSE, 1998, "The Endogeneity of the Optimum Currency Area Criteria", Economic Journal, vol. 108 (449), pp. 1009-1025.

GAlAt, G., HEALTH, A. et P. McGUIRE, 2007, "Mise en évidence du carry trade", Rapport trimestriel, Banque des règlements internationaux.

GANSER, D., RENTSCH, H. et U. WAGSCHAL, 2003, Cavalier seul. La Suisse 10 ans après le non à l'EEE, Avenir Suisse, Zürich, Editions Labor et Fides.

GENBERG, H., 2000, “ Switzerland and European Monetary Integration: Challenges and Options ", National Research Program, Foundations and Possibilities of Swiss Foreign Policy, Berne, September.

GOSH, A. R., GULDE, A.-M., OSTRY, J. D. et H. C. WOLF, 1997, "Does the nominal exchange rate regime matter?", NBER working paper 5874.

HAUSMANN, R., GAVIN, M., PAGES-SERRA, C. et E. STEIN, 1999, "Financial Turmoil and the Choice of Exchange Rate Regime”, working paper 400, Inter-American Development Bank.

KRUGMAN, P., 1991, "The Move toward Free Trade Zones”, Economic Review, Federal Reserve Bank of Kansas City, pp. 7-58.

LARDIC, S. et V. MIGNON, 2002, Économétrie des séries temporelles macroéconomiques et financières, Economica.

LEVY-YEYATI, E. et F. STURZENEGGER, 2003, “To Float or To Fix: Evidence on the Impact of Exchange Rate Regimes on Growth", American Economic Review, vol. 93 (4), pp. 1173-1193. 
OBSTFELD, M. et K. ROGOFF, 1995, "Exchange Rate Dynamics Redux", Journal of Political Economy, vol. 103 (3), pp. 624-660.

PLIHON, D. et C. GUILLAUMIN, 2008, "Les transformations du système monétaire international", Questions Internationales, 34, pp. 6-18.

RANALDO, A. et P. SÖDERLIND, 2007, "Safe Haven Currencies", Discussion Paper 22, Universität St-Gallen.

REINHART, C. et K. ROGOFF, 2004, "The Modern History of Exchange Rate Arrangements: A Reinterpretation”, Quarterly Journal of Economics, vol. 119 (1), pp. 1-48.

SCHWOK, R., 2006, Suisse-Union européenne, l'adhésion impossible?, Presses polytechniques et universitaires romandes, Collection Le savoir suisse.

SFREDDO, C., 2002, "Pourquoi les taux d'intérêt réels sont-ils si bas en Suisse ?", Institut de macroéconomie appliquée, Créa, Ecole des Hautes études commerciales, Lausanne, pp. 1-37. STULZ, J., 2007, "Exchange rate pass-through in Switzerland: Evidence from vector autoregressions", Swiss National Bank Economic Studies.

UBS, 2000, "The Future of the Swiss Franc. Independence or clinging to the euro boat?", UBS Group Economic Research Studies. 